\title{
Cardioprotective role of S-nitrosylated hemoglobin from rbc
}

\author{
Claude A. Piantadosi \\ Duke University Medical Center, Durham, North Carolina, USA.
}

\begin{abstract}
Nitric oxide (NO) is a potent mediator of blood vessel dilation and is released by several cell sources. Red blood cells (rbc) release NO when hemoglobin that has been S-nitrosylated at Cys93 of the $\beta$-chain ( $\beta$ Cys93) transitions from the oxygenated form to the deoxygenated form. This transition occurs in response to reduced tissue oxygenation and is an important physiologic regulator of hypoxic vasodilation. In this issue of the $\mathrm{JCl}$, Zhang and colleagues demonstrate that S-nitrosylation of hemoglobin at $\beta$ Cys93 is important for tissue oxygenation after cardiac injury. Mice harboring mutations that prevent S-nitrosylation of $\beta$ Cys93 had higher rates of morbidity and mortality following cardiac injury compared with WT; however, adaptive cardiac vascularization was increased in some mutant mice and reduced cardiac injury in these animals. The results of this study reveal a previously unexplored role of $S$-nitrosylated hemoglobin in cardioprotection.
\end{abstract}

\section{The rbc influence on coronary circulation}

The red blood cell $(\mathrm{rbc})$ dilates peripheral blood vessels during episodic local hypoxia, and this capacity is mediated by the release of low-molecular weight vasodilators, one of which conveys nitric oxide (NO) bioactivity to the blood vessel from $S$-nitrosohemoglobin (SNO-Hb). SNO-Hb releases NO activity allosterically when hemoglobin transitions from the $\mathrm{R}$ (oxygenated) to the $\mathrm{T}$ (deoxygenated) state, thereby improving local vasodilation during $\mathrm{O}_{2}$ transport to cells. SNO-Hb is found chiefly on the hemoglobin $\beta$-chain at the highly conserved $\beta C y s 93$ residue, and approximately 1 in every $1,000 \beta$-chains carries a molecule of NO that is released as lowmolecular weight SNO, for instance, on thiol compounds such as cysteinyl-NO (CSNO) or glutathionyl-NO (GSNO), to facilitate rbcmediated vasodilation. A study by Zhang et al. in this issue reveals that the loss of $\beta$ Cys 93 SNO-Hb results in highly detrimental consequences and impairs rbc blood-flow regulation in myocardial infarction (MI) and ven- tricular pressure overload in mice. Together, these results indicate that this rbc system has a compelling influence on the coronary circulation (1).

Physiologically, rbc-mediated vasodilation is a characteristic of native rbc, which are able to signal changes in oxygen tension $\left(\mathrm{PO}_{2}\right)$ through graded vasodilator and vasoconstrictor activity that pairs the vasoactive responses of intact blood vessels with changes in tissue $\mathrm{O}_{2}$ availability. Thus, $\mathrm{NO}$ is carried by rbc in the circulation, together with $\mathrm{O}_{2}$ and $\mathrm{CO}_{2}$, as a respiratory gas. In this paradigm, $\mathrm{SNO}-\mathrm{Hb}$ supports tissue oxygenation during periods of high $\mathrm{O}_{2}$ demand or during hypoxemia or ischemia by facilitating the microvascular delivery and distribution of $\mathrm{O}_{2}$ to respiring cells (2).

Despite strong proof-of-principle that $\mathrm{SNO}-\mathrm{Hb}$ is important for tissue oxygenation under physiological conditions, the effect of $\mathrm{SNO}-\mathrm{Hb}$ dysfunction in disease models is not well understood, even though low SNO-Hb levels have been implicated in the vascular problems of diabetes, sickle

Related Article: p. 4654

Conflict of interest: The author has declared that no conflict of interest exists.

Reference information: / Clin Invest. 2016;126(12):4402-4403. doi:10.1172/JCI91303.

cell anemia, congestive heart failure, pulmonary hypertension, and preeclampsia and in the adverse effects of blood transfusions (2). Detection of the physiological effects specific to SNO-Hb is complicated by the fact that endothelial NO synthase (NOS3) also participates in blood flow regulation and that endothelial dysfunction interferes with NO-dependent vasodilation. Moreover, rbc SNO-Hb derives in part from NOS3 (3), which up to now, has made it difficult for specific NO species to be assigned as the cause for defects in tissue blood flow regulation.

Any definitive test to detect $\mathrm{SNO}-\mathrm{Hb}-$ specific contributions requires a physiologically accurate mouse model, in which murine $\mathrm{Hb}$ is replaced with human $\mathrm{Hb}$. The generation of such mice was technically challenging because the human $\mathrm{Hb}$ has only one $\beta$-chain while the mouse $\mathrm{Hb}$ has two $\beta$-chains (major and minor). A humanized $\beta$ Cys93-null $\mathrm{Hb}$ mouse containing a $\beta$ Cys93Ala substitution was constructed at the University of Alabama at Birmingham by Townes and reported by Isbell et al. in 2008 (4). In characterizing these mice, Isbell et al. specified that they could detect no role for $\beta$ Cys 93 in hypoxic vasodilation; however, their measurements $\mathrm{O}_{2}$-binding curves, cardiac output, blood pressure, and exercise tolerance) lacked the resolution needed to detect the SNO-Hb-specific microcirculatory physiology of tissue $\mathrm{O}_{2}$ transfer during hypoxia.

In 2015, Zhang and colleagues of the Stamler laboratory at Case Western Reserve finally detected defects in blood flow and tissue oxygenation in the mice lacking $\beta$ Cys93 (5). In that work, mice lacking the $\beta$ C93 SNO-binding site (some mice also contained $\sim 10 \%$ human fetal $\alpha_{2} \gamma_{2}$ tetramers to improve fetal survivability) were found by analyzing $\mathrm{Hb}$-bound $\mathrm{NO}$ and shown to have absolute SNO-Hb levels that were similar to those of WT mice; however, hypoxic unloading of the rbc SNO pool in the absence of $\beta$ Cys 93 was far less than in WT mice and lacked 
clear allosteric SNO-Hb kinetics. Additionally, animals lacking $\beta$ Cys 93 displayed significantly increased fetal mortality, and live animals subsequently developed myocardial ischemia and cardiovascular decompensation and displayed greater mortality during short-term hypoxia, thus supporting the modern view of a three-gas respiratory cycle.

\section{The role of SNO-Hb in cardiac injury}

The fundamental findings of the Stamler group in 2015, which demonstrated that mice with the $\beta$ Cys93A mutation had increased MI and cardiogenic shock under hypoxia, also implied that rbc operating through allosterically coupled SNO-Hb activity play an important protective role in the heart that could have a meaningful impact on ischemic or hypertensive heart disease and heart failure. The Stamler group now reports, in mouse models of heart disease, lack of SNO at $\beta$ Cys93 enhances cardiac injury and overall mortality after left coronary artery occlusion-induced MI and following pressure overload-induced cardiac hypertrophy in the transverse aortic constriction (TAC) model (1).
Interestingly, some of the $\beta$ Cys93mutant mice exhibited the capacity to increase cardiac vascular collaterals via an unknown mechanism, and mice with this adaptation developed less ischemic injury after MI and had a better outcome. For instance, in the absence of collateralization, $18 \%$ of $\gamma \beta C 93$ control mice died after $\mathrm{MI}$, but in the $\beta \mathrm{C} 93 \mathrm{~A}$ (and $\gamma \beta C 93 \mathrm{~A}$ ) strains, more than $50 \%$ of the mice died. The TAC model produced similarly higher mortality rates in the $\beta C 93 \mathrm{~A}$ strains; however, mutant mice that survived longer than five days after TAC showed reduced ejection fractions, fractional shortening, and cardiac output as well as increased dilation of the left ventricle compared with WT mice after four weeks.

\section{Conclusions}

The finding by Zhang et al. that the presence of $\mathrm{Hb} \beta \mathrm{C} 93$ mitigates both ischemic and pressure overload-induced heart damage demonstrates the importance of rbcderived SNO-based vasodilation in cardiac protection. By implication, rbc SNO-Hb levels might prove to be a useful biomarker of human cardiac disease or cardiovascular risk. Additionally, manipulation of SNO-
$\mathrm{Hb}$ may have potential as a therapeutic target to improve tissue oxygenation in cardiovascular states of compromise.

\section{Acknowledgments}

This work was supported by two grants from the NIH: R01-AI095424 and P01 HL108801 (to C. Piantodosi).

Address correspondence to: Claude A. Piantadosi, Duke University Medical Center, Box 3315, Duke South Hospital, Durham, North Carolina 27710, USA. Phone: 919.684.6143; E-mail: claude.piantadosi@ duke.edu.

1. Zhang R, et al. Hemoglobin S-nitrosylation plays an essential role in cardioprotection. J Clin Invest. 2016;126(12):4654-4658.

2. Allen BW, Stamler JS, Piantadosi CA. Hemoglobin, nitric oxide and molecular mechanisms of hypoxic vasodilation. Trends Mol Med. 2009;15(10):452-460.

3. Michel T, Vanhoutte PM. Cellular signaling and NO production. Pflugers Arch. 2010;459(6):807-816.

4. Isbell TS, et al. SNO-hemoglobin is not essential for red blood cell-dependent hypoxic vasodilation. Nat Med. 2008;14(7):773-777.

5. Zhang R, et al. Hemoglobin $\beta$ Cys 93 is essential for cardiovascular function and integrated response to hypoxia. Proc Natl Acad Sci US A. 2015;112(20):6425-6430. 